\title{
Identities for generalized balancing numbers
}

\author{
Robert Frontczak* \\ Landesbank Baden-Württemberg \\ Am Hauptbahnhof 2, 70173 Stuttgart, Germany \\ e-mail: robert. frontczak@lbbw. de
}

Received: 25 July 2018

Revised: 21 December 2018

Accepted: 19 January 2019

\begin{abstract}
We study properties of generalized balancing numbers. We start with some basic identities. Thereafter, we focus on connections to generalized Fibonacci numbers. Using generating functions we prove fundamental relations between these two sequences. Many interesting examples involving balancing, Lucas-balancing, Fibonacci, and Lucas numbers are obtained as special cases of our relations.
\end{abstract}

Keywords: Generating function, Balancing number, Fibonacci number.

2010 Mathematics Subject Classification: 11B37, 11B39.

\section{Introduction}

In 1999, Behera and Panda [1] introduced a new sequence of numbers called balancing numbers. According to the authors, a positive integer $x$ is a balancing number with balancer $r$, if it is the solution to the Diophantine equation

$$
1+2+\cdots+(x-1)=(x+1)+(x+2)+\cdots+(x+r) .
$$

The set of balancing numbers is usually denoted by $\left(B_{n}\right)_{n \geq 0}$. Behera and Panda [1] derived interesting properties of these numbers. Among other results, they showed that $B_{n}$ satisfies the following recurrence relation of second order

$$
B_{n+1}=6 B_{n}-B_{n-1}, \quad n \geq 1,
$$

* Disclaimer: Statements and conclusions made in this article are entirely those of the author. They do not necessarily reflect the views of LBBW. 
with initial terms $B_{0}=0$ and $B_{1}=1$. Another result about balancing numbers is, that $B_{n}$ is a balancing number, if and only if $B_{n}^{2}$ is a triangular number, i.e., $8 B_{n}^{2}+1$ is a perfect square. Another interesting result from [1] is that the three functions

$$
y_{1}(x)=2 x \sqrt{8 x^{2}+1}, \quad y_{2}(x)=3 x+\sqrt{8 x^{2}+1} \quad \text { and } \quad y_{3}(x)=17 x+6 \sqrt{8 x^{2}+1},
$$

also generate balancing numbers, i.e., $y_{i}\left(B_{n}\right)(i=1,2,3)$ is a balancing number. The sequence $C_{n}=\sqrt{8 B_{n}^{2}+1}$ is called a Lucas-balancing number. It satisfies the same recurrence relation as $B_{n}$,

$$
C_{n+1}=6 C_{n}-C_{n-1}, \quad n \geq 1,
$$

with initial terms $C_{0}=1$ and $C_{1}=3 .\left(B_{n}\right)_{n \geq 0}$ is sequence A001109 in the OEIS [16], whereas $\left(C_{n}\right)_{n \geq 0}$ has the id-number A001541 in OEIS.

The sequences $B_{n}$ and $C_{n}$ have been studied extensively in recent years. We refer to the references $[3,7,8,11-15]$. Some properties are similar to those for Fibonacci numbers. For instance, the Catalan identities are given by ([3])

$$
B_{n+r} B_{n-r}-B_{n}^{2}=-B_{r}^{2} \quad \text { and } \quad C_{n+r} C_{n-r}-C_{n}^{2}=C_{r}^{2}-1 .
$$

For $r=1$, the Catalan identities reduce to the Cassini identities:

$$
B_{n+1} B_{n-1}-B_{n}^{2}=-1 \quad \text { and } \quad C_{n+1} C_{n-1}-C_{n}^{2}=8 .
$$

A famous result concerning balancing numbers was obtained by Liptai in [8], where he proved that there is no Fibonacci number within the set of balancing numbers except 1 .

Generalizations of balancing numbers can be obtained in various ways (see [9] or [10]). In the present paper we will take equations (1.1) and (1.2) as special examples of the more general definition

$$
b_{n+1}=6 b_{n}-b_{n-1}, \quad n \geq 1,
$$

with $b_{0}=\alpha$ and $b_{1}=\beta$. We assume that $\alpha$ and $\beta$ are fixed integers with $|\alpha|+|\beta|>0$. The sequence $\left(b_{n}(\alpha, \beta)\right)_{n \geq 0}$ will be called a generalized balancing sequence. Obviously, $b_{n}(0,1)=$ $B_{n}$ and $b_{n}(1,3)=C_{n}$. For notational ease, we will write almost persistently $b_{n}$ for $b_{n}(\alpha, \beta)$. Finally, we remark that the $b_{n}$ as defined above are not balancing in the sense of [2].

In this article we prove many interesting properties of generalized balancing numbers. We start with some basic identities. Thereafter, we focus on connections to generalized Fibonacci numbers. Using generating functions we prove fundamental relations between these two fascinating sequences.

\section{Basic properties of generalized balancing numbers $b_{n}$}

Generating functions will play a key role in our proofs. For that reason, we start with the generating functions for $b_{n}$. This approach can be also found in the articles [14] and [15] to establish identities involving balancing and Lucas-balancing numbers. 
Proposition 2.1. The ordinary generating function for $\left(b_{n}\right)_{n \geq 0}$ is given by

$$
f_{b_{n}}(x)=\frac{\alpha+(-6 \alpha+\beta) x}{1-6 x+x^{2}}
$$

Also, we have

$$
f_{b_{2 n}}(x)=\frac{\alpha+(-35 \alpha+6 \beta) x}{1-34 x+x^{2}}
$$

and

$$
f_{b_{2 n+1}}(x)=\frac{\beta+(-6 \alpha+\beta) x}{1-34 x+x^{2}} .
$$

Proof. The first result is obvious and follows from the definition and

$$
f_{b_{n}}(x)-6 x f_{b_{n}}(x)+x^{2} f_{b_{n}}(x)=\alpha+(-6 \alpha+\beta) x .
$$

The other statements follow from Lemma 2.2 in [4].

Using the generating function for $b_{n}$, it is straightforward to derive their Binet form. We have

$$
b_{n}(\alpha, \beta)=X \lambda_{1}^{n}-Y \lambda_{2}^{n},
$$

with

$$
X=\frac{\beta-\alpha \lambda_{2}}{\lambda_{1}-\lambda_{2}} \quad \text { and } \quad Y=\frac{\beta-\alpha \lambda_{1}}{\lambda_{1}-\lambda_{2}},
$$

where $\lambda_{1}=3+\sqrt{8}$ and $\lambda_{2}=3-\sqrt{8}$. The constants $\lambda_{1,2}$ are solutions to $1-6 x+x^{2}=0$. We note that the Binet form can be utilized to extend the definition to negative indices:

$$
b_{-n}(\alpha, \beta)=X \lambda_{1}^{-n}-Y \lambda_{2}^{-n}=-\left(Y \lambda_{1}^{n}-X \lambda_{2}^{n}\right) .
$$

Observe that for $b_{n}=B_{n}$ we have $X=Y=\frac{1}{\lambda_{1}-\lambda_{2}}$ and $B_{-n}=-B_{n}$. Similarly, for $b_{n}=C_{n}$ we have $X=-Y=\frac{1}{2}$ and $C_{-n}=C_{n}$.

A direct consequence of Proposition 2.1 are the following relations:

Corollary 2.2. For each $n \geq 0$ we have

$$
\begin{gathered}
C_{n}=B_{n+1}-3 B_{n}, \\
6 C_{2 n}=B_{2 n+2}-17 B_{2 n},
\end{gathered}
$$

and

$$
C_{2 n+1}+C_{2 n-1}=3\left(B_{2 n+1}-B_{2 n-1}\right) .
$$

Proof. The equations follow from

$$
\begin{aligned}
x f_{C_{n}}(x) & =(1-3 x) f_{B_{n}}(x), \\
6 x f_{C_{2 n}}(x) & =(1-17 x) f_{B_{2 n}}(x),
\end{aligned}
$$

and

$$
(1+x) f_{C_{2 n+1}}(x)=(3-3 x) f_{B_{2 n+1}}(x)
$$


Proposition 2.3. Catalan's and Cassini's identities for $b_{n}$ are given by

$$
b_{n+r} \cdot b_{n-r}-b_{n}^{2}=-X Y\left(\lambda_{1}^{r}-\lambda_{2}^{r}\right)^{2} .
$$

Especially,

$$
b_{n+1} \cdot b_{n-1}-b_{n}^{2}=-32 X Y \text {. }
$$

Proof. We have

$$
\begin{aligned}
b_{n+r} \cdot b_{n-r}-b_{n}^{2} & =\left(X \lambda_{1}^{n+r}-Y \lambda_{2}^{n+r}\right)\left(X \lambda_{1}^{n-r}-Y \lambda_{2}^{n-r}\right)-\left(X \lambda_{1}^{n}-Y \lambda_{2}^{n}\right)^{2} \\
& =2 X Y-X Y\left(\lambda_{1}^{2 r}+\lambda_{2}^{2 r}\right) \\
& =-X Y\left(\lambda_{1}^{2 r}+\lambda_{2}^{2 r}-2\left(\lambda_{1} \lambda_{2}\right)^{r}\right) \\
& =-X Y\left(\lambda_{1}^{r}-\lambda_{2}^{r}\right)^{2} .
\end{aligned}
$$

Next, we state two combinatorial formulas for $b_{n}$.

Proposition 2.4. For each generalized balancing number $b_{n}$ it holds that

$$
b_{n}=\alpha \sum_{k=0}^{\left\lfloor\frac{n}{2}\right\rfloor}(-1)^{k}\left(\begin{array}{c}
n-k \\
k
\end{array}\right) 6^{n-2 k}+(-6 \alpha+\beta) \sum_{k=0}^{\left\lfloor\frac{n-1}{2}\right\rfloor}(-1)^{k}\left(\begin{array}{c}
n-1-k \\
k
\end{array}\right) 6^{n-1-2 k}
$$

Proof. From (2.1) we obtain

$$
f_{b_{n}}(x)=(\alpha+(-6 \alpha+\beta) x) \sum_{n=0}^{\infty}\left(6 x-x^{2}\right)^{n} .
$$

Expand the term in brackets binomially, and the proof is completed.

The case $b_{n}=B_{n}$ appears as Theorem 3.1 in [14]. When $b_{n}=C_{n}$, then the above identity turns into

$$
C_{n}=\sum_{k=0}^{\left\lfloor\frac{n}{2}\right\rfloor}(-1)^{k}\left(\begin{array}{c}
n-k \\
k
\end{array}\right) 6^{n-2 k}-3 \sum_{k=0}^{\left\lfloor\frac{n-1}{2}\right\rfloor}(-1)^{k}\left(\begin{array}{c}
n-1-k \\
k
\end{array}\right) 6^{n-1-2 k} .
$$

Obviously, from (2.2) and (2.3) similar identities for $b_{2 n}$ and $b_{2 n+1}$ can be derived. The next result generalizes Theorem 3.3 from [14].

Proposition 2.5. For each generalized balancing number $b_{n}$ it holds that

$$
b_{n}=(X+Y) \sum_{k=1}^{\left\lfloor\frac{n+1}{2}\right\rfloor}\left(\begin{array}{c}
n \\
2 k-1
\end{array}\right) 3^{n+1-2 k}(\sqrt{8})^{2 k-1}+(X-Y) \sum_{k=0}^{\left\lfloor\frac{n}{2}\right\rfloor}\left(\begin{array}{c}
n \\
2 k
\end{array}\right) 3^{n-2 k} \cdot 8^{k}
$$

Proof. As in [14], we set $\lambda_{1}=a+b$ and $\lambda_{2}=a-b$ with $a=3$ and $b=\sqrt{8}$. This produces

$$
b_{n}=X(a+b)^{n}-Y(a-b)^{n}=\sum_{k=0}^{n}\left(\begin{array}{l}
n \\
k
\end{array}\right) a^{n-k} b^{k}\left(X-Y(-1)^{k}\right) .
$$

Now, split the sum into even and odd parts and the proof is completed. 
Proposition 2.6. For each $n \geq 0$ we have the following identities:

$$
\begin{gathered}
\sum_{k=0}^{2 n}\left(\begin{array}{c}
2 n \\
k
\end{array}\right) b_{k}=8^{n} b_{n}, \\
\sum_{k=0}^{2 n}\left(\begin{array}{c}
2 n \\
k
\end{array}\right)(-1)^{k} b_{k}=4^{n} b_{n}, \\
\sum_{k=0}^{n}\left(\begin{array}{c}
2 n \\
2 k
\end{array}\right) b_{2 k}=\left(2^{3 n-1}+2^{2 n-1}\right) b_{n},
\end{gathered}
$$

and for $n \geq 1$

$$
\sum_{k=0}^{\left\lfloor\frac{2 n-1}{2}\right\rfloor}\left(\begin{array}{c}
2 n \\
2 k+1
\end{array}\right) b_{2 k+1}=\left(2^{3 n-1}-2^{2 n-1}\right) b_{n}
$$

Proof. The essential part in the proof is to observe that $\left(\lambda_{1,2}+1\right)^{2}=8 \lambda_{1,2}$ and $\left(\lambda_{1,2}-1\right)^{2}=4 \lambda_{1,2}$. This, in combination with the binomial theorem, proves the first two equations. The third identity follows from adding the first two equations, whereas the last identity is obtained by subtracting these.

The next result provides us with a first convolution identity for generalized balancing numbers. The identity may be proved directly using the Binet form.

Theorem 2.7. Let $l$ and $m$ be integers. Then it holds that

$$
\begin{aligned}
\sum_{k=0}^{n} b_{m k} \cdot b_{l n-m k}= & (n+1)\left(X^{2} \lambda_{1}^{l n}+Y^{2} \lambda_{2}^{l n}\right) \\
& -\frac{X Y}{\lambda_{1}^{m}-\lambda_{2}^{m}}\left(\lambda_{1}^{n(2 m-l)+m}-\lambda_{2}^{n(2 m-l)+m}+\lambda_{1}^{l n+m}-\lambda_{2}^{l n+m}\right) .
\end{aligned}
$$

Especially, for $l=m$ we have

$$
\sum_{k=0}^{n} b_{m k} \cdot b_{m(n-k)}=(n+1)\left(X^{2} \lambda_{1}^{m n}+Y^{2} \lambda_{2}^{m n}\right)-\frac{2 X Y}{\lambda_{1}^{m}-\lambda_{2}^{m}}\left(\lambda_{1}^{m(n+1)}-\lambda_{2}^{m(n+1)}\right) .
$$

Proof. By the Binet formula with $\lambda_{1} \lambda_{2}=1$ we can calculate

$$
\begin{aligned}
\sum_{k=0}^{n} b_{m k} \cdot b_{l n-m k}= & \sum_{k=0}^{n}\left(X \lambda_{1}^{m k}-Y \lambda_{2}^{m k}\right)\left(X \lambda_{1}^{l n-m k}-Y \lambda_{2}^{l n-m k}\right) \\
= & (n+1)\left(X^{2} \lambda_{1}^{l n}+Y^{2} \lambda_{2}^{l n}\right) \\
& -X Y \sum_{k=0}^{n}\left(\lambda_{2}^{l n}\left(\frac{\lambda_{1}}{\lambda_{2}}\right)^{m k}+\lambda_{1}^{l n}\left(\frac{\lambda_{2}}{\lambda_{1}}\right)^{m k}\right) .
\end{aligned}
$$


Using the geometric series and simplifying yields

$$
\begin{aligned}
\sum_{k=0}^{n} b_{m k} \cdot b_{l n-m k}= & (n+1)\left(X^{2} \lambda_{1}^{l n}+Y^{2} \lambda_{2}^{l n}\right) \\
& -X Y\left(\frac{\lambda_{1}^{m(n+1)}-\lambda_{2}^{m(n+1)}}{\lambda_{1}^{m}-\lambda_{2}^{m}}\left(\frac{1}{\lambda_{2}^{n(m-l)}}+\frac{1}{\lambda_{1}^{n(m-l)}}\right)\right) \\
= & (n+1)\left(X^{2} \lambda_{1}^{l n}+Y^{2} \lambda_{2}^{l n}\right) \\
& -\frac{X Y}{\lambda_{1}^{m}-\lambda_{2}^{m}}\left(\lambda_{1}^{m(n+1)}-\lambda_{2}^{m(n+1)}\right)\left(\lambda_{1}^{n(m-l)}+\lambda_{2}^{n(m-l)}\right)
\end{aligned}
$$

Expanding the terms in parentheses proves the formula.

For $l=m=1$ and $b_{n}=B_{n}$ the formula reduces to

$$
\sum_{k=0}^{n} B_{k} B_{n-k}=\frac{1}{16}\left((n+1) C_{n}-B_{n+1}\right),
$$

and for $b_{n}=C_{n}$ we obtain

$$
\sum_{k=0}^{n} C_{k} C_{n-k}=\frac{1}{2}\left((n+1) C_{n}+B_{n+1}\right) .
$$

Similarly, for $l=m=2$

$$
\sum_{k=0}^{n} B_{2 k} B_{2(n-k)}=\frac{1}{16}\left((n+1) C_{2 n}-\frac{1}{6} B_{2 n+2}\right),
$$

and

$$
\sum_{k=0}^{n} C_{2 k} C_{2(n-k)}=\frac{1}{2}\left((n+1) C_{2 n}+\frac{1}{6} B_{2 n+2}\right) .
$$

Generating functions are a prominent tool to prove different kinds of convolution sums. See for instance [4], [5], and [6]. We give two advanced examples. Hereby, we focus on convolution sums containing $b_{2 n}$ and $b_{2 n+1}$. To abbreviate notation, we set $\alpha^{*}=-6 \alpha+\beta$ and $\beta^{*}=-35 \alpha+6 \beta$. The first result we would like to present is the following (symmetric) identity:

Theorem 2.8. For $n \geq 0$ we have the following convolution:

$$
\begin{gathered}
\sum_{k=0}^{n} b_{2 k}\left(\beta^{2} b_{2(n+2-k)}+2 \alpha^{*} \beta b_{2(n+1-k)}+\alpha^{* 2} b_{2(n-k)}\right)+b_{2 n+2}\left(b_{2} \beta^{2}+2 \alpha \alpha^{*} \beta\right)+\alpha \beta^{2} b_{2 n+4} \\
=\sum_{k=0}^{n} b_{2 k+1}\left(\alpha^{2} b_{2(n+2-k)+1}+2 \alpha \beta^{*} b_{2(n+1-k)+1}+\beta^{* 2} b_{2(n-k)+1}\right) \\
+b_{2 n+3}\left(b_{3} \alpha^{2}+2 \alpha \beta \beta^{*}\right)+\alpha^{2} \beta b_{2 n+5} .
\end{gathered}
$$

Proof. From (2.2) and (2.3) we deduce the relation

$$
\left(\beta+\alpha^{*} x\right)^{2} f_{b_{2 n}}^{2}(x)=\left(\alpha+\beta^{*} x\right)^{2} f_{b_{2 n+1}}^{2}(x) .
$$


Expanding and writing it as a power series, we see that the LHS equals

$$
L H S=\beta^{2} \sum_{n=0}^{\infty} \sum_{k=0}^{n} b_{2 k} b_{2(n-k)} x^{n}+2 \alpha^{*} \beta \sum_{n=1}^{\infty} \sum_{k=0}^{n-1} b_{2 k} b_{2(n-1-k)} x^{n}+\alpha^{* 2} \sum_{n=2}^{\infty} \sum_{k=0}^{n-2} b_{2 k} b_{2(n-2-k)} x^{n} .
$$

The RHS is given by

$$
\begin{gathered}
\text { RHS }=\alpha^{2} \sum_{n=0}^{\infty} \sum_{k=0}^{n} b_{2 k+1} b_{2(n-k)+1} x^{n}+2 \alpha \beta^{*} \sum_{n=1}^{\infty} \sum_{k=0}^{n-1} b_{2 k+1} b_{2(n-1-k)+1} x^{n} \\
+\beta^{* 2} \sum_{n=2}^{\infty} \sum_{k=0}^{n-2} b_{2 k+1} b_{2(n-2-k)+1} x^{n} .
\end{gathered}
$$

The identity follows by gathering terms, comparing the coefficients of $x^{n}$, and replacing $n$ by $n+2$.

When $b_{n}=B_{n}$, then the formula reduces to

$$
36 \sum_{k=0}^{n} B_{2 k+1} B_{2(n-k)+1}=6 B_{2 n+2}+\sum_{k=0}^{n} B_{2 k}\left(B_{2(n+2-k)}+2 B_{2(n+1-k)}+B_{2(n-k)}\right) .
$$

When $b_{n}=C_{n}$, then the convolution becomes

$$
\begin{aligned}
& \sum_{k=0}^{n} C_{2 k}\left(C_{2(n+2-k)}-2 C_{2(n+1-k)}+C_{2(n-k)}\right)+15 C_{2 n+2}+C_{2 n+4} \\
= & \frac{1}{9} \sum_{k=0}^{n} C_{2 k+1}\left(C_{2(n+2-k)+1}-34 C_{2(n+1-k)+1}+289 C_{2(n-k)+1}\right)+\frac{1}{3}\left(C_{2 n+5}-C_{2 n+3}\right) .
\end{aligned}
$$

Theorem 2.9. For $n \geq 0$ we have the following convolution:

$$
\begin{gathered}
\sum_{k=0}^{n} b_{2 k+1}\left((-\alpha+6 \beta) b_{2(n+2-k)+1}-2 \alpha b_{2(n+1-k)+1}-\beta^{*} b_{2(n-k)+1}\right) \\
+b_{2 n+3}\left(b_{3}(-\alpha+6 \beta)-2 \alpha \beta\right)+b_{2 n+5} \beta(-\alpha+6 \beta) \\
=\beta^{2}(n+3) b_{2 n+6}+2 \alpha^{*} \beta(n+2) b_{2 n+4}+\alpha^{* 2}(n+1) b_{2 n+2} .
\end{gathered}
$$

Proof. Again, from (2.2) and (2.3) we derive the relation

$$
\left(\beta+\alpha^{*} x\right)^{2} f_{b_{2 n}}^{\prime}(x)=\left((-\alpha+6 \beta)-2 \alpha x-\beta^{*} x^{2}\right) f_{b_{2 n+1}}^{2}(x),
$$

where $f_{b_{2 n}}^{\prime}(x)$ is the first derivative with respect to $x$. The remaining part of the proof is as in the last theorem. We omit the lengthy details.

When $b_{n}=B_{n}$, then the formula simplifies to

$$
\begin{aligned}
& \sum_{k=0}^{n} B_{2 k+1}\left(B_{2(n+2-k)+1}-B_{2(n-k)+1}\right) \\
= & \frac{1}{6}\left((n+3) B_{2 n+6}+2(n+2) B_{2 n+4}+(n+1) B_{2 n+2}\right)-35 B_{2 n+3}-B_{2 n+5} .
\end{aligned}
$$


When $b_{n}=C_{n}$, then the convolution becomes

$$
\begin{aligned}
& \sum_{k=0}^{n} C_{2 k+1}\left(17 C_{2(n+2-k)+1}-2 C_{2(n+1-k)+1}+17 C_{2(n-k)+1}\right) \\
= & 9(n+3) C_{2 n+6}-18(n+2) C_{2 n+4}+9(n+1) C_{2 n+2}-51 C_{2 n+5}-1677 C_{2 n+3} .
\end{aligned}
$$

Equations (2.2) and (2.3) allow to produce more results of this kind. We leave it to the interested reader.

\section{Connections to generalized Fibonacci numbers}

This section contains new fundamental relations between balancing and Fibonacci numbers. Analogous results for Fibonacci and Tribonacci numbers have been established recently in [5] and [6]. As usual, let $\left(u_{n}\right)_{n \geq 0}$ be a generalized Fibonacci sequence, i.e.,

$$
u_{n}=u_{n-1}+u_{n-2}, \quad n \geq 2,
$$

where $u_{0}$ and $u_{1}$ are arbitrary numbers not both being zero. When $u_{0}=0$ and $u_{1}=1$, then $u_{n}=F_{n}$ is the Fibonacci sequence (A000045 in the OEIS [16]) and when $u_{0}=2$ and $u_{1}=1$, then $u_{n}=L_{n}$ is the Lucas sequence (A000032 in [16]). Our first result is the following theorem.

Theorem 3.1. Let $\left(b_{n}\right)_{n \geq 0}$ and $\left(u_{n}\right)_{n \geq 0}$ denote the generalized balancing and Fibonacci sequences, respectively. Then, for each $n \geq 2$, we have the following identity:

$$
u_{0} b_{n}+\left(u_{1}-u_{0}\right) b_{n-1}=\alpha u_{n}+(\beta-\alpha) u_{n-1}+\sum_{k=0}^{n-2} u_{k}\left(5 b_{n-1-k}-2 b_{n-2-k}\right) .
$$

Proof. The generating function for $\left(u_{n}\right)_{n \geq 0}$ is given by

$$
f_{u_{n}}(x)=\frac{u_{0}+\left(u_{1}-u_{0}\right) x}{1-x-x^{2}} .
$$

Now, notice that

$$
1-6 x+x^{2}=1-x-x^{2}-5 x+2 x^{2}=\frac{u_{0}+\left(u_{1}-u_{0}\right) x-5 x f_{u_{n}}(x)+2 x^{2} f_{u_{n}}(x)}{f_{u_{n}}(x)} .
$$

Hence, using the generating function (2.1)

$$
\left(u_{0}+\left(u_{1}-u_{0}\right) x-5 x f_{u_{n}}(x)+2 x^{2} f_{u_{n}}(x)\right) f_{b_{n}}(x)=(\alpha+(-6 \alpha+\beta) x) f_{u_{n}}(x) .
$$

For the LHS we have

$$
\begin{aligned}
\text { LHS }= & u_{0} \sum_{n=0}^{\infty} b_{n} x^{n}+\left(u_{1}-u_{0}\right) \sum_{n=1}^{\infty} b_{n-1} x^{n}-5 \sum_{n=1}^{\infty} \sum_{k=0}^{n-1} u_{k} b_{n-1-k} x^{n} \\
& +2 \sum_{n=2}^{\infty} \sum_{k=0}^{n-2} u_{k} b_{n-2-k} x^{n} .
\end{aligned}
$$


The RHS equals

$$
R H S=\alpha \sum_{n=0}^{\infty} u_{n} x^{n}+(-6 \alpha+\beta) \sum_{n=1}^{\infty} u_{n-1} x^{n} .
$$

Comparing the coefficients of $x^{n}$ gives the statement.

As special cases of Theorem 3.1 we state explicit results for the pairs $\left(b_{n}=B_{n}, u_{n}=F_{n}\right)$, $\left(b_{n}=B_{n}, u_{n}=L_{n}\right),\left(b_{n}=C_{n}, u_{n}=F_{n}\right)$, and $\left(b_{n}=C_{n}, u_{n}=L_{n}\right)$. When $b_{n}=B_{n}$ and $u_{n}=F_{n}$, then equation (3.2) simplifies to

$$
B_{n}=F_{n}+\sum_{k=0}^{n-1} F_{k}\left(5 B_{n-k}-2 B_{n-1-k}\right) .
$$

When $b_{n}=B_{n}$ and $u_{n}=L_{n}$, then equation (3.2) becomes

$$
2 B_{n}=B_{n-1}+L_{n-1}+\sum_{k=0}^{n-2} L_{k}\left(5 B_{n-1-k}-2 B_{n-2-k}\right) .
$$

When $b_{n}=C_{n}$ and $u_{n}=F_{n}$, then we get

$$
C_{n}=F_{n+1}+2 F_{n}+\sum_{k=0}^{n-1} F_{k}\left(5 C_{n-k}-2 C_{n-1-k}\right) .
$$

Finally, when $b_{n}=C_{n}$ and $u_{n}=L_{n}$, then

$$
2 C_{n}=C_{n-1}+L_{n}+2 L_{n-1}+\sum_{k=0}^{n-2} L_{k}\left(5 C_{n-1-k}-2 C_{n-2-k}\right) .
$$

Our next theorem deals with even indexed numbers $b_{2 n}$ and $u_{2 n}$.

Theorem 3.2. For $n \geq 1$ the following identity holds:

$$
u_{0} b_{2 n}+\left(u_{1}-2 u_{0}\right) b_{2 n-2}-\alpha u_{2 n}+(35 \alpha-6 \beta) u_{2 n-2}=31 \sum_{k=0}^{n-1} u_{2 k} b_{2(n-1-k)} .
$$

Proof. The generating function for $\left(u_{2 n}\right)_{n \geq 0}$ is given by (see for instance [6])

$$
f_{u_{2 n}}(x)=\frac{u_{0}+\left(u_{1}-2 u_{0}\right) x}{1-3 x+x^{2}} .
$$

Comparing this function with the generating function (2.2) and using similar arguments as in the previous proof, we can derive the following relation:

$$
\left(u_{0}+\left(u_{1}-2 u_{0}\right) x-31 x f_{u_{2 n}}(x)\right) f_{b_{2 n}}(x)=(\alpha+(-35 \alpha+6 \beta) x) f_{u_{2 n}}(x) .
$$

Comparing the coefficients of $x^{n}$ establishes the identity.

As specific examples of the theorem we can state the following mixed convolution identities: 
1. $b_{2 n}=B_{2 n}$ and $u_{2 n}=F_{2 n}$ :

$$
\sum_{k=0}^{n} F_{2 k} B_{2(n-k)}=\frac{1}{31}\left(B_{2 n}-6 F_{2 n}\right)
$$

2. $b_{2 n}=B_{2 n}$ and $u_{2 n}=L_{2 n}$ :

$$
\sum_{k=0}^{n} L_{2 k} B_{2(n-k)}=\frac{1}{31}\left(2 B_{2 n+2}-3 B_{2 n}-6 L_{2 n}\right) .
$$

3. $b_{2 n}=C_{2 n}$ and $u_{2 n}=F_{2 n}$ :

$$
\sum_{k=0}^{n} F_{2 k} C_{2(n-k)}=\frac{1}{31}\left(C_{2 n}-F_{2 n+2}+17 F_{2 n}\right) .
$$

4. $b_{2 n}=C_{2 n}$ and $u_{2 n}=L_{2 n}$ :

$$
\sum_{k=0}^{n} L_{2 k} C_{2(n-k)}=\frac{1}{31}\left(2 C_{2 n+2}-3 C_{2 n}-L_{2 n+2}+17 L_{2 n}\right) .
$$

The next theorem contains relations for the odd indexed numbers $b_{2 n+1}$ and $u_{2 n+1}$.

Theorem 3.3. For $n \geq 1$ the following identity holds:

$$
u_{1} b_{2 n+1}+\left(u_{0}-u_{1}\right) b_{2 n-1}-\beta u_{2 n+1}+(6 \alpha-\beta) u_{2 n-1}=31 \sum_{k=0}^{n-1} u_{2 k+1} b_{2(n-1-k)+1} .
$$

Proof. The generating function for $\left(u_{2 n+1}\right)_{n \geq 0}$ is (see for instance [6])

$$
f_{u_{2 n+1}}(x)=\frac{u_{1}+\left(u_{0}-u_{1}\right) x}{1-3 x+x^{2}} .
$$

In view of the generating function (2.3) we get the relation:

$$
\left(u_{1}+\left(u_{0}-u_{1}\right) x-31 x f_{u_{2 n+1}}(x)\right) f_{b_{2 n+1}}(x)=(\beta+(-6 \alpha+\beta) x) f_{u_{2 n+1}}(x) .
$$

Again, comparing the coefficients of $x^{n}$ proves the desired identity.

As examples we get the following set of mixed convolutions involving odd indices:

1. $b_{2 n+1}=B_{2 n+1}$ and $u_{2 n+1}=F_{2 n+1}$ :

$$
\sum_{k=0}^{n} F_{2 k+1} B_{2(n-k)+1}=\frac{1}{31}\left(B_{2 n+3}-B_{2 n+1}-F_{2 n+3}-F_{2 n+1}\right) .
$$

2. $b_{2 n+1}=B_{2 n+1}$ and $u_{2 n+1}=L_{2 n+1}$ :

$$
\sum_{k=0}^{n} L_{2 k+1} B_{2(n-k)+1}=\frac{1}{31}\left(B_{2 n+3}+B_{2 n+1}-L_{2 n+3}-L_{2 n+1}\right) .
$$


3. $b_{2 n+1}=C_{2 n+1}$ and $u_{2 n+1}=F_{2 n+1}$ :

$$
\sum_{k=0}^{n} F_{2 k+1} C_{2(n-k)+1}=\frac{1}{31}\left(C_{2 n+3}-C_{2 n+1}-3 F_{2 n+3}+3 F_{2 n+1}\right) .
$$

4. $b_{2 n+1}=C_{2 n+1}$ and $u_{2 n+1}=L_{2 n+1}$ :

$$
\sum_{k=0}^{n} L_{2 k+1} C_{2(n-k)+1}=\frac{1}{31}\left(C_{2 n+3}+C_{2 n+1}-3 L_{2 n+3}+3 L_{2 n+1}\right) .
$$

Moreover, adding (3.19) and (3.20) gives

$$
\sum_{k=0}^{n}\left(F_{2 k+1}+L_{2 k+1}\right) B_{2(n-k)+1}=\frac{1}{31}\left(2 B_{2 n+3}-\left(F_{2 n+3}+L_{2 n+3}\right)-\left(F_{2 n+1}+L_{2 n+1}\right)\right) .
$$

In a similar manner, we get

$$
\sum_{k=0}^{n}\left(F_{2 k+1}+L_{2 k+1}\right) C_{2(n-k)+1}=\frac{1}{31}\left(2 C_{2 n+3}-3\left(F_{2 n+3}+L_{2 n+3}\right)+3\left(F_{2 n+1}+L_{2 n+1}\right)\right) .
$$

Two additional identities can be obtained by subtracting the above equations.

We point out that the six generating functions for $b_{n}, b_{2 n}, b_{2 n+1}, u_{n}, u_{2 n}$, and $u_{2 n+1}$ can be combined in a few more ways to easily produce more results of this nature. We provide three additional relations without proof.

Theorem 3.4. For $n \geq 1$ the following identity holds:

$$
u_{0} b_{n}+\left(u_{1}-2 u_{0}\right) b_{n-1}-\alpha u_{2 n}+(6 \alpha-\beta) u_{2 n-2}=3 \sum_{k=0}^{n-1} u_{2 k} b_{n-1-k} .
$$

Theorem 3.5. For $n \geq 1$ the following identity holds:

$$
u_{1} b_{n}+\left(u_{0}-u_{1}\right) b_{n-1}-\alpha u_{2 n+1}+(6 \alpha-\beta) u_{2 n-1}=3 \sum_{k=0}^{n-1} u_{2 k+1} b_{n-1-k} .
$$

Theorem 3.6. For $n \geq 1$ the following identity holds:

$$
u_{1} b_{2 n}+\left(u_{0}-u_{1}\right) b_{2 n-2}-\alpha u_{2 n+1}+(35 \alpha-6 \beta) u_{2 n-1}=31 \sum_{k=0}^{n-1} u_{2 k+1} b_{2(n-1-k)} .
$$

From these relations we get

$$
\begin{gathered}
\sum_{k=0}^{n} F_{2 k} B_{n-k}=\frac{1}{3}\left(B_{n}-F_{2 n}\right), \\
\sum_{k=0}^{n} F_{2 k+1} B_{n-k}=\frac{1}{3}\left(B_{n+1}-B_{n}-F_{2 n+1}\right)
\end{gathered}
$$

and

$$
\sum_{k=0}^{n} F_{2 k+1} B_{2(n-k)}=\frac{1}{31}\left(B_{2 n+2}-B_{2 n}-6 F_{2 n+1}\right) .
$$

More specific examples are left to the interested reader. 


\section{References}

[1] Behera, A., \& Panda, G. K. (1999). On the square roots of triangular numbers, The Fibonacci Quart., 37 (2), 98-105.

[2] Berczes, A., Liptai, K., \& Pink, I. (2010). On generalized balancing sequences, The Fibonacci Quart., 48, 121-128.

[3] Catarino, P., Campos, H., \& Vasco, P. (2015). On some identities for balancing and cobalancing numbers, Anal. Math. et Inf., 45, 11-24.

[4] Frontczak, R. (2018). Convolutions for Generalized Tribonacci Numbers and Related Results, Int. J. Math. Anal., 12 (7), 307-324.

[5] Frontczak, R. (2018). Some Fibonacci-Lucas-Tribonacci-Lucas Identities, The Fibonacci Quart., 56 (3), 263-274.

[6] Frontczak, R. (2019). Relations for Generalized Fibonacci and Tribonacci Sequences, Notes on Number Theory and Discrete Mathematics, 25 (1), 178-192.

[7] Keskin, R. \& Karaatli, O. (2012). Some new properties of balancing numbers and square triangular numbers, J. Integer Seq., 15, Article 12.1.4.

[8] Liptai, K. (2004). Fibonacci balancing numbers, The Fibonacci Quart., 42, 330-340.

[9] Liptai, K., Luca, F., Pintér, A. \& Szalay, L. (2009). Generalized balancing numbers, Indag. Math. (N.S.), 20, 87-100.

[10] Özkoc, A. (2015). Tridigonal Matrices via $k$-Balancing Number, British J. of Math. Comput. Sci., $10(4), 1-11$.

[11] Panda, G. K., Komatsu, T., \& Davala, R. K. (2018). Reciprocal Sums of Sequences Involving Balancing and Lucas-Balancing Numbers, Math. Reports, 20, 201-214.

[12] Ray, P. K. (2014). Some congruences for balancing and Lucas-balancing numbers and their applications, Integers, 14, \#A8.

[13] Ray, P. K. (2015) Balancing and Lucas-balancing sums by matrix methods, Math. Reports, 17 (2), 225-233.

[14] Ray, P. K., Patel, S. \& Mandal, M. (2016). Identities for balancing numbers using generating function and some new congruence relations, Notes on Number Theory and Discrete Mathematics, 22 (4), 41-48.

[15] Ray, P. K., \& Sahu, J. (2016). Generating functions for certain balancing and Lucasbalancing numbers, Palestine J. Math., 5 (2), 122-129.

[16] Sloane, N. J. A. The On-Line Encyclopedia of Integer Sequences, Published electronically at https: //oeis.org. 